\title{
Low-level Laser Therapy with Novel Array of Light Source and Individualized Program for Treatment of Androgenetic Alopecia: A 16-week, Randomized, Double-blind, Sham Device-controlled Study
}

\author{
Jee-Woo Kim¹ \\ Yeo-Seon Kwon² \\ Yoon-Young Chang ${ }^{3}$ \\ Sung-Ho Hong ${ }^{3}$ \\ Jung-Won Shin ${ }^{1}$ \\ Jung-Im $\mathrm{Na}^{1}$ \\ Chang-Hun Huh'
}

\footnotetext{
${ }^{1}$ Department of Dermatology, Seoul National University Bundang Hospital, Seongnam, Korea ${ }^{2}$ Home Entertainment Company, LG Electronics, Seoul, Korea

${ }^{3}$ Beauty/Medical Task, E\&M center, CTO, LG Electronics, Seoul, Korea
}

\begin{abstract}
Background and Objectives
Low-level laser therapy (LLLT) is used widely to promote hair growth in androgenetic alopecia (AGA). This study examined the clinical efficacy and safety of a home-use LLLT device with a newly designed array of light sources and software optimized for individual types of AGA.

\section{Materials and Methods}

The study was a randomized, double-blind, sham device-controlled trial. Forty-eight subjects (39 men and nine women) were assigned randomly in a 2:1 ratio to use either the test device (LG Pra'L HGN1, LG electronics, Koreal or sham device. The subjects used the LLLT device three times a week for 16 weeks. Phototrichogram was used to measure the hair density and hair thickness at 0,8 , and 16-weeks. Adverse events were closely monitored.
\end{abstract}

\section{Results}

After 16 weeks of using the device, the test group showed a significant increase in hair density and hair thickness compared to the control. In the test group, the hair density increased 6.96 counts $/ \mathrm{cm}^{2}$ at eight weeks and 13.67 counts $/ \mathrm{cm}^{2}$ at 16 weeks from the baseline. The hair thickness increased $7.21 \mu \mathrm{m}$ at eight weeks and $11.80 \mu \mathrm{m}$ at 16 weeks compared to the baseline.

\section{Conclusion}

The home-use LLLT device with a novel array of light sources and an individualized program according to the types of hair loss appears to be an effective and safe treatment modality for both male and female AGA patients.

\section{Key words}

Low-level laser therapy; Light emitting diode; Photobiomodulation; Androgenetic alopecia; Phototrichogram

\footnotetext{
Correspondence

Chang-Hun Huh

Department of Dermatology, Seoul National University Bundang Hospital, 166 Gumi-ro, Bundang-gu, Seongnam 13620, Korea

Tel.: +82-31-787-7313

Fax: +82-2-787-4058

E-mail: chhuh@snu.ac.kr

(C) Korean Society for Laser Medicine and Surgery

(c) This is an open access article distributed under the terms of the Creative Commons Attribution NonCommercial License (http://creativecommons.org/ licenses/by-nc/4.0) which permits unrestricted noncommercial use, distribution, and reproduction in any medium, provided the original work is properly cited.
}

A




\section{INTRODUCTION}

Hair loss has been known to cause significant distress in both men and women, which impairs individual's quality of life in various apsects. ${ }^{1,2}$ Androgenetic alopecia (AGA) is the most common type of hair loss in men, which occurs in genetically susceptible individuals under the influence of androgens. AGA is characterized by follicular miniaturization in a distinctive pattern of hair loss. It usually appears in the third and fourth decades, while adolescent AGA starts after puberty between 13.5 and 15 years of age. ${ }^{3.4}$ Pathophysiologic features of AGA include alteration of hair cycle, follicular miniaturization, and inflammation. ${ }^{5}$ Topical minoxidil and oral medication, such as finasteride, have been approved by Food and Drug Administration for the treatment of AGA.

Lately, several documents have been reported, which aim to investigate the therapeutic role of low-level laser device in AGA. ${ }^{6-8}$ Low-level laser therapy (LLLT) utilizes non-thermal effect of red or near-infrared laser light in a low intensity, which promote tissue repair and stimulate cellular activities. It is also termed as photobiomodulation or photobiostimulation. Beneficial and therapeutic effects of LLLT has been recruited in a wide range of medical situations, including wound healing, nerve regeneration, pain control, and alopecia. ${ }^{6,9}$

\section{MATERIALS AND METHODS}

\section{Study design}

A clinical study was designed as a randomized, doubleblind, sham device-controlled trial at a single research center (Seoul National University Bundang Hospital,
Seongnam, Korea). Study protocol was approved by the Institution Review Board of the research center and Ministry of Food and Drug Safety of Korea (E-1909/564-002).

\section{Patient enrollment}

Forty-eight healthy volunteers aged 19 to 65 were recruited. Prior to participation in the study, each subject provided an Informed consent and they were screened to verify that they met the inclusion and exclusion criteria for the study. All subjects had androgenetic hair loss. (Norwood-Hamilton classification of lla to $\mathrm{V}$ for men or Ludwig classification of I to II for women, or BASP classification specific type V1, V2, F1, and F2) and Fitzpatrick skin type I to IV. Subjects were excluded if they had taken and treated following medications that might affect hair growth and loss within 6 months prior to first visit and during the study: finasteride, cyclosporine, minoxidil, dutasteride. Medical history was collected and physical examinations of subjects were performed. Subjects who have chronic dermatologic conditions le.g., eczema, psoriasis, infection), photosensitive conditions on skins, a history of keloid formation or poor wound healing, a history of human immunodeficiency virus infections, a history drug or alcohol abuse, and have pacemaker, defibrillator, or other active implantable devices. Subject who have tattoo and hair weave also excluded.

\section{Test devices}

The Pra.L HGN1 (LG Electronics, Seoul, Korea) is a LLLT (3R-Laser level, Illa) device with a light source consisting of light-emitting diodes (LEDs; 104 units), 5 mW pulsed laser diodes (LDs; 50 units) and $50 \mathrm{~mW}$ pulsed LDs assembled with a waveguide (16 units) (Fig. 1). The
A

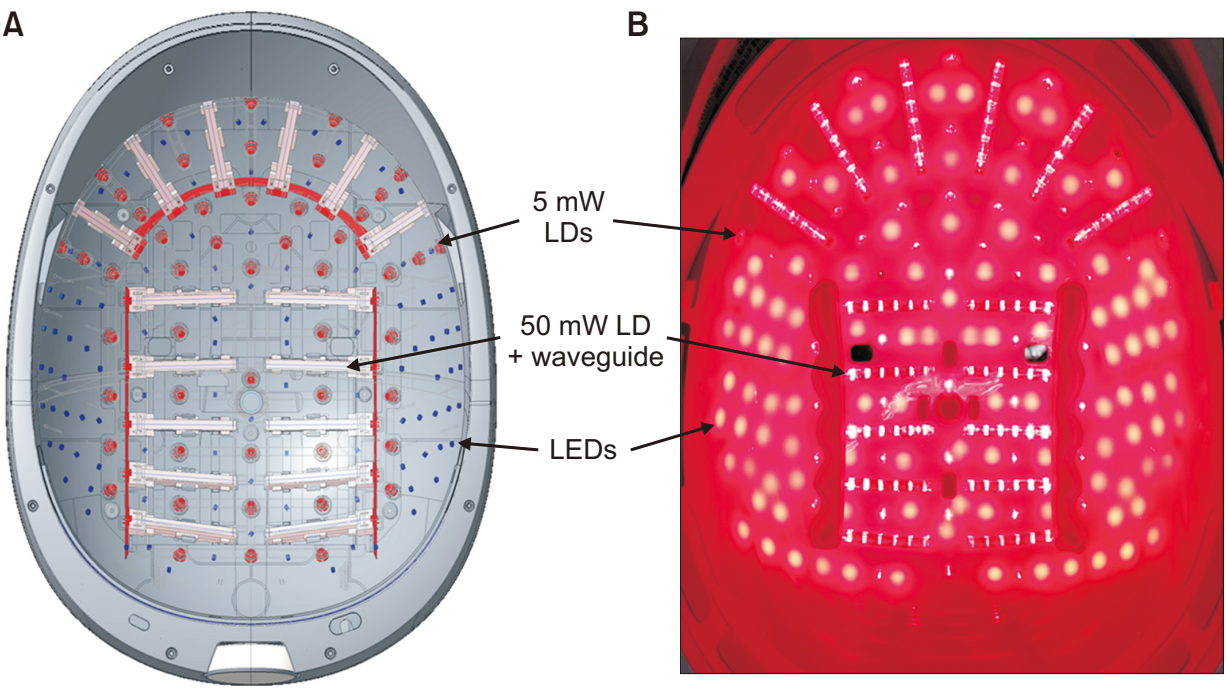

Fig. 1. (A) Image of $L G$ Pra' $L$ HGN1. Inside layout of LG Pra'L HGN1 device with light source consisting of LED (104 units), laser diodes (50 units), and laser diodes with a waveguide (16 units). (B) Real image of 250 red light sources when the device is operating. 
waveguide manufactured by the plastic injection-mold process is distributed to the light output surface via the process of passing one light generated from the LD through the primary and secondary reflective surfaces. The secondary reflective surface is designed to distribute the light reflected from the primary reflective surface into six (Fig. 2).

The emitting wavelength is $630 \pm 20 \mathrm{~nm}$ in LED (CTSRZ12A, SEOULVIOSYS, Ansan, Korea), $655 \pm 5 \mathrm{~nm}$ in $5 \mathrm{~mW}$ LD (QL65D6SA, QSI Co., Ltd, Cheonan, Koreal and 660 \pm 7 $\mathrm{nm}$ in $50 \mathrm{~mW}$ LD (QL65J7SA, QSI Co., Ltd). All of the LDs run pulsed light through $250 \mathrm{~Hz}$.

The mean energies per unit of each light source with USB2000 (Spectrometer and optical fiber; Ocean optics, Largo, FL, USA) for LEDs and PM400+S120C (Optical Power and Energy Meter Console + photodiode power sensor, Thorlabs GmbH, Bergkirchen, Germany) for LDs were $3.04 \mathrm{~mW} / \mathrm{cm}^{2}$ for $630 \mathrm{~nm}$ LED, $2.99 \mathrm{~mW}$ for $655 \mathrm{~nm}$ LD, and $3.08 \mathrm{~mW} / \mathrm{spot}$ for $660 \mathrm{~nm}$ LD with waveguide.

The sham device was identical in appearance and its regulator operated, although it emitted white LED light. We randomly assigned all of the subjects who satisfied the inclusion and exclusion criteria to the LLLT or sham device group, and they were totally blinded. Subjects were instructed on how to operate each device at the baseline visit.

\section{Randomization and masking}

Subjects were randomized into experimental and control group according to random sequence using the complete randomization method. Each group using the test devices or sham devices were contained in sealed boxes recorded with a serial number in order of registration. During whole process, double-blind status was maintained for both investigators and subjects.

\section{Study design (protocol)}

To analyze hair growth, an area was selected in vertex zone of the scalp of each subject. The hairs in the selected area were cut to a maximum height of $3 \mathrm{~mm}$ and in area at about $1 \mathrm{~cm}$ in diameter. The distance between LED or $L D$ and scalp reaches almost $1 \mathrm{~cm}$ in maximum, although slight variations may exist according to specific area of scalp such as vertex, temporal or occiput. The area was marked with a tattoo using black ink in aseptic condition.

Each subject was assigned in a 2:1 ratio to use either LG Pra.L HGN1 or a sham device. Subjects were instructed how use to an assigned device from blinded investigator and used it themselves at home for 27 minutes and 3 times per week. During one session of intervention with 27 minutes, total energy released from test device was calculated to be $3.3 \mathrm{~J} / \mathrm{cm}^{2}$. The study duration was 16 weeks, and monitored at 0,8 , and 16 weeks after the first visit. Subjects and investigators remained totally blinded until study completion.

\section{Efficacy evaluation}

Primary efficacy endpoint was change in hair density in the target area at 16 weeks from baseline. Hair density and thickness were evaluated with phototrichogram using Folliscope (LeadM, Seoul, Korea) at baseline, 8-week, and 16 -week. Phototrichogram images were taken in a
A

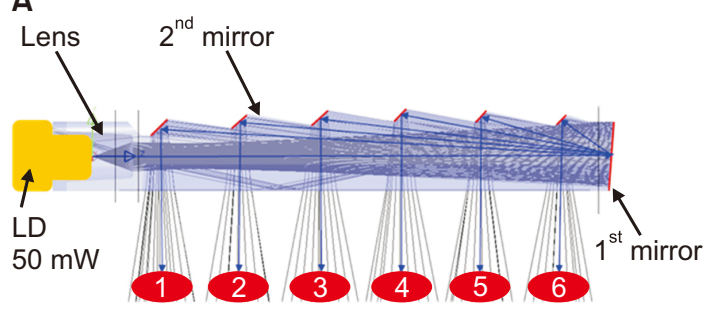

B

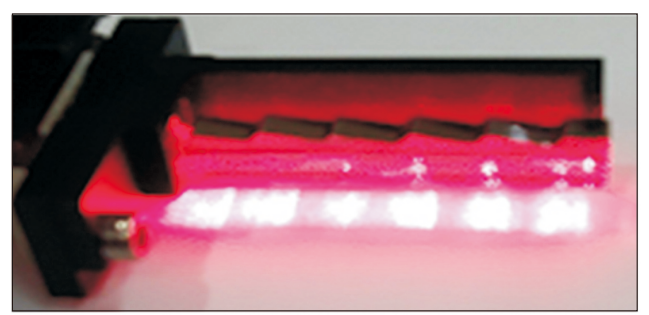

C

1)

2)

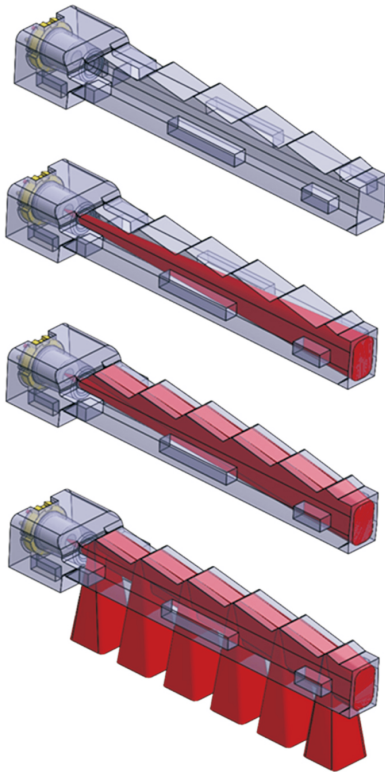

Fig. 2. (A) Novel array of light sources with a waveguide. Schematic view of laser diode assembled waveguide. The waveguide distributed to 6 light sources via the process of passing one light generated from the laser diode through the primary and secondary reflective surfaces. (B) Actual image of light through a waveguide. (C) Laser light pathway via waveguide. 1) Laser off, 2) Laser on 1st mirror, 3) 1st mirror 2nd mirror, 4) 2nd mirror Scalp. 
central location with tattoos, with 100-fold magnification lens. The image was analyzed and calculated the counts per unit area and the hair thickness using Folliscope 5.0 software (LeadM) by blinded investigators.

Global photographic assessment was evaluated using customized photographic device. The customized photograph device is designed to take pictures of the top, the front, and the sides of both sides at a fixed angle of each subject's head. Global photographs were taken at baseline, 8-week, and 16-week in a space with constant brightness. The global photographs were evaluated by three independent investigator to the degree of improvement, based on 5-point rating method (Excellent, Good, Slight, No growth, and Worse).

Subjects answered questionnaires for self-assessment of improvement and satisfaction of hair growth at the 8 -week and 16-week visits. The degree of improvement of hair regrowth by subjects was assessed using a $100 \mathrm{~mm}$ visual analog scale (VAS). The higher the VAS score, the higher the degree of improvement.
All adverse events were collected from investigator and subjects during the study period. Investigator observed the scalp condition of subjects at every visits, and the subjects also informed the investigator of their abnormal cases.

\section{Statistical analysis}

After the end of the study, statistical analysis was conducted using SAS Software (version 9.4; Cary, NC, USA). At the base point and 16 weeks after the treatment, we analyzed amount of change compared to base using a covariance analysis method (ANCOVA) with a covariate for the base value. Treatment site anomalies were classified by body organ class (SOC) criteria in MedDRA (Ver.23.0), and Fisher's exact test was used for the analysis of adherence and adverse reactions. All statistical analyses were two-sided at a $5 \%$ level of significance.

Table 1. Study population and characteristics of the subjects at baseline.

\begin{tabular}{|c|c|c|c|c|}
\hline & LG Pra'L HGN1 (N=32) & Sham device $(\mathrm{N}=16)$ & Total $(\mathrm{N}=48)$ & $p$-value \\
\hline Gender & & & & 0.2380 \\
\hline Male (\%) & $24(75.00)$ & $15(93.75)$ & $39(81.25)$ & \\
\hline Female $(\%)$ & $8(25.00)$ & $1(6.25)$ & $9(18.75)$ & \\
\hline Age (years), mean (SD) & $42.06(8.93)$ & $46.69(7.74)$ & $43.60(8.75)$ & 0.0842 \\
\hline Height $(\mathrm{cm})$, mean (SD) & $171.05(10.07)$ & $170.39(6.43)$ & $170.83(8.96)$ & 0.5995 \\
\hline Weight $(\mathrm{kg})$, mean $(\mathrm{SD})$ & $74.61(12.52)$ & $71.39(8.43)$ & $73.53(11.33)$ & 0.3592 \\
\hline \multicolumn{5}{|c|}{ Norwood-Hamilton classification, $\mathrm{N}(\%)$} \\
\hline Total N & 17 & 14 & 31 & \\
\hline$\| \mathrm{A}$ & $5(29.41)$ & $1(7.14)$ & $6(19.35)$ & \\
\hline III & $0(0.00)$ & $1(7.14)$ & $1(3.23)$ & \\
\hline III Vertex & $5(29.41)$ & $7(50.00)$ & $12(38.71)$ & \\
\hline III A & $0(0.00)$ & $0(0.00)$ & $0(0.00)$ & \\
\hline IV & $3(17.65)$ & $4(28.57)$ & $7(22.58)$ & \\
\hline IV A & $0(0.00)$ & $0(0.00)$ & $0(0.00)$ & \\
\hline V & $4(23.53)$ & $1(7.14)$ & $5(16.13)$ & \\
\hline \multicolumn{5}{|l|}{ Ludwig classification, N (\%) } \\
\hline Total N & 7 & 1 & 8 & \\
\hline Type I & $7(100.00)$ & $1(100.00)$ & $8(100.00)$ & \\
\hline Type II & $0(0.00)$ & $0(0.00)$ & $0(0.00)$ & \\
\hline \multicolumn{5}{|l|}{ BASP classification, $\mathrm{N}(\%)$} \\
\hline Total N & 18 & 9 & 27 & \\
\hline V1 & 8 (44.44) & $2(22.22)$ & $10(37.04)$ & \\
\hline V2 & $2(11.11)$ & $5(55.56)$ & $7(25.93)$ & \\
\hline $\mathrm{F} 1$ & $6(33.33)$ & $1(11.11)$ & $7(25.93)$ & \\
\hline \multirow[t]{2}{*}{ F2 } & $2(11.11)$ & $1(11.11)$ & $3(11.11)$ & \\
\hline & LG Pra'L HGN1 (N=30) & Sham device $(\mathrm{N}=16)$ & Total $(\mathrm{N}=46)$ & \\
\hline Hair density (hairs $/ \mathrm{cm}^{2}$ ), mean (SD) & $77.23(17.34)$ & $95.56(18.56)$ & $83.61(19.66)$ & \\
\hline Hair thickness $(\mu \mathrm{m})$, mean (SD) & $77.66(15.09)$ & $76.94(18.31)$ & $76.76(16.08)$ & \\
\hline
\end{tabular}

$\mathrm{SD}$, standard deviation. 


\section{RESULTS}

\section{Study population}

The total 48 subjects registered for the study, of which 39 subjects were men and 9 subjects were women. 32 subjects are assigned in test group and used LG Pra. L HGN1, and the other 16 subjects are control group and used sham device. 2 subjects were excluded from per-protocol analysis. One was dropped out of the study by having a perm that might affect hair analysis, while another subject was excluded with less than $70 \%$ compliance. There were no statistically significant differences between two groups in age, gender, height, and weight (All $p>0.05$; Table 1).

\section{Primary efficacy analysis (per-protocol set)}

After 16 weeks using LG Pra.L HGN1, the test group had significantly improved on hair density and hair thickness compared to group using sham device by phototricogram l $p<0.0001$ and $p<0.0001$, hair density and thickness respectively) (Fig. 3). In the group using LG HGN1, the mean change of hair density increased $6.95 \pm 1.14$ counts $/ \mathrm{cm}^{2}$ at 8 weeks and $13.67 \pm 1.20$ counts $/ \mathrm{cm}^{2}$ at 16 weeks from baseline. On the other hand, control group using sham device decreased by $0.21 \pm 1.62 \mathrm{count} / \mathrm{cm}^{2}$ at 8 weeks and by $3.19 \pm 1.71$ counts $/ \mathrm{cm}^{2}$ at 16 weeks from baseline. The differences in hair density between the test and control group were 16.86 hairs $/ \mathrm{cm}^{2}(21.64 \%)$ at 16 weeks.

In the group using LG HGN1, mean change of hair thickness increased $7.21 \pm 1.83 \mu \mathrm{m}$ at 8 weeks, and 11.80 $\pm 1.67 \mu \mathrm{m}$ at 16 weeks compared to baseline. Mean change of hair thickness in control group decreased 0.94 $\pm 2.50 \mu \mathrm{m}$ at 8 weeks and $3.45 \pm 2.29 \mu \mathrm{m}$ at 16 weeks
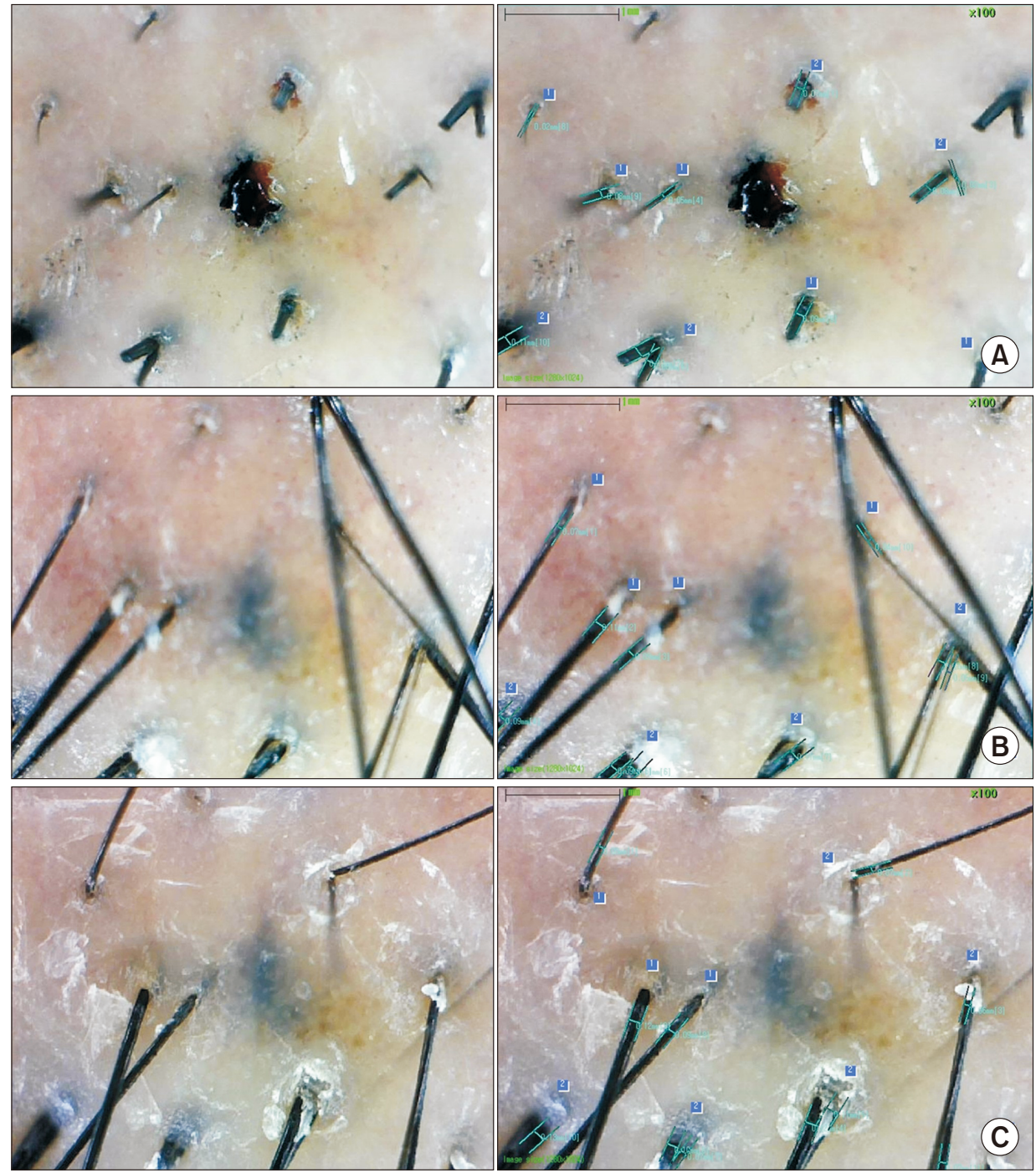

Fig. 3. Phototrichogram images taken in a central location with 100 fold magnification at (A) baseline, (B) 8-week, and (C) 16-week of LG Pra'L HGN1-treated subject (R-0148). The images were analyzed and calculated by Folliscope 5.0 software (Lead M, Seoul, Korea), which are shown on the right side of each row of images. 
from baseline. The differences in hair thickness between the test and control group were $15.26 \mu \mathrm{m}(19.46 \%)$ at 16 weeks.

\section{Secondary efficacy analysis (per-protocol set)}

Global photographic analysis for hair regrowth after 8 weeks and 16 weeks compared to baseline by three independent investigators are summarized in Fig. 4A. After 16 weeks in test group, 1 person (3.33\%) was rated "good improved", 12 subjects (40\%) were rated "slightly improved", and 16 subjects (53.33\%) were rated "no growth". On the other hand, 8 subjects (50\%) were rated "slightly improved" and 8 subjects (50\%) were rated "no growth" in the control group. In the global photographic analysis, the test group showed slightly higher improvement rates, but differences between two groups were not statistically significant ( $p=0.8978$ ) (Fig. 5). The images of subjects evalu- ated as "slightly improved" is shown in Fig. 6 and "good improved" is shown in Fig. 7.

Self-assessment for hair regrowth after 8 weeks and 16 weeks by subjects are demonstrated in Fig. 4B. The VAS score from baseline to 16 weeks in self-assessment was 54.47 VAS in test group versus 45.56 VAS in control group. The VAS score at 8-week in self-assessment was 40.47 VAS in test group and 30.63 VAS in control group. However, differences between two groups were not statistically significant $(p=0.3154)$.

\section{Safety evaluation}

There was no serious adverse event occurred in any subjects. Two subjects (6.25\%) in test group were experienced abnormal cases in the area of treatment at least once during study. All of the abnormal cases in test group were about itching in the scalp. These subjects sponta-
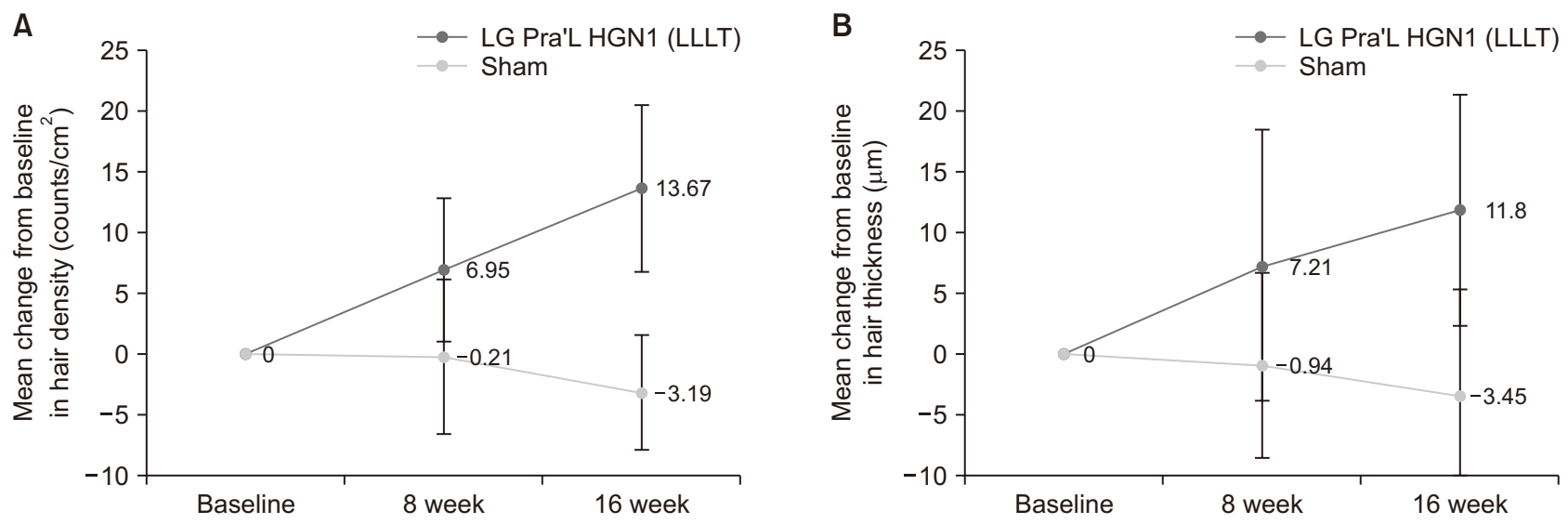

Fig. 4. The mean changes from baseline in hair density (a) and hair thickness (b) at 8 week and 16 week after treatment of LG Pra'L HGN1 and sham devices.
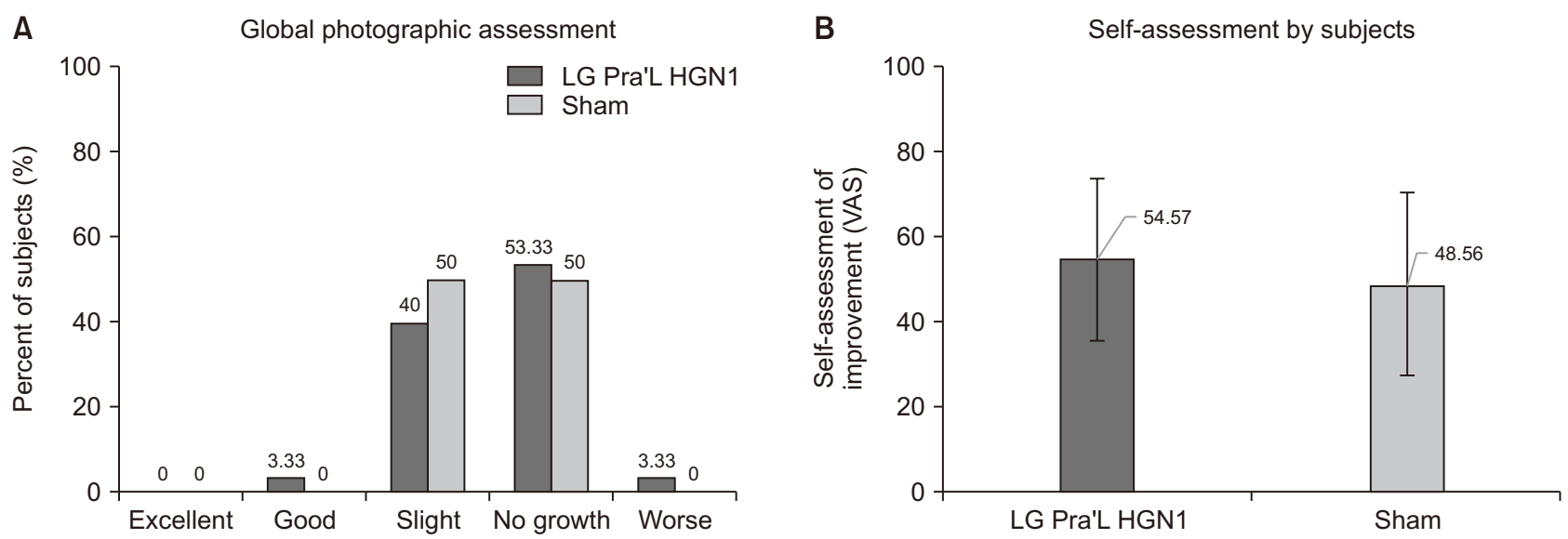

Fig. 5. The global photographic assessment at 16-week compared to baseline by three independent investigator (A) and self-assessment at 16-week compared to baseline subjects (B). 


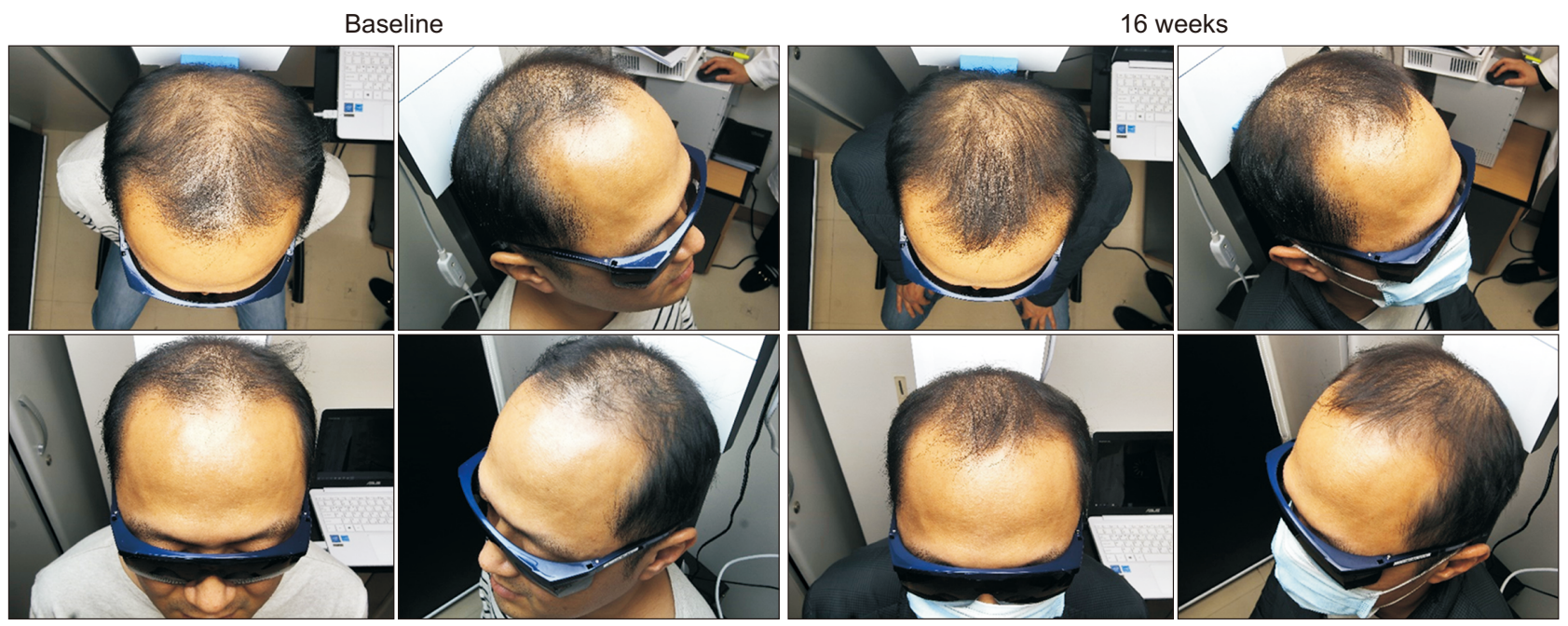

Fig. 6. Global photographic images with top, front, right side, left side angles of baseline and 16-week of LG Pra'L HGN1-treated subject (R-0113) who was rated as "slightly improved" by three independent dermatologists.
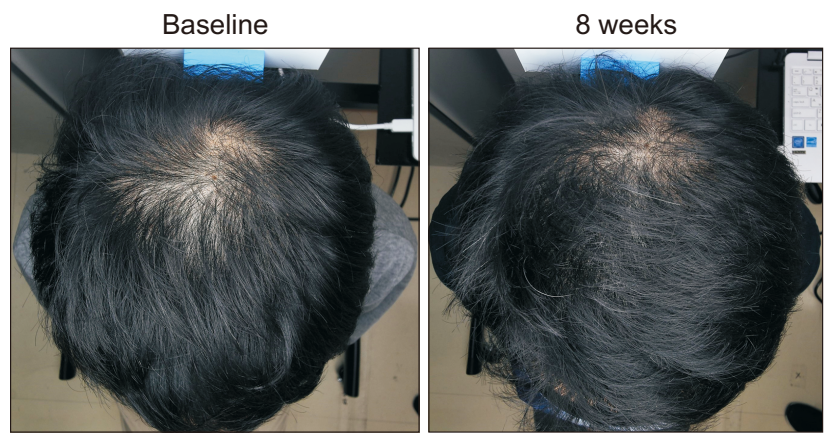

neously resolved within the period of study without and aftereffects. Another two subjects (6.25\%) in test group were complained abnormal cases in a non-treatment area; one subject complained of eye disorder (asthenopia) and the other felt dizzy. As these adverse events were closely monitored, they gradually decreased in terms of severity and frequency, which didn't require any further examinations. All of these abnormal cases in nontreatment area were confirmed as non-serious adverse event, and these subjects recovered without any further treatment and aftereffect.

\section{DISCUSSION}

Androgenetic alopecia is the most common form of non-scarring alopecia in men and women. So far, treatments of AGA with highest levels of evidence include topical minoxidil solution and oral 5-alpha reductase inhinitors. ${ }^{10}$ LLLT has recently gained attention in research fields for treatment of AGA, though LLLT for photobio-

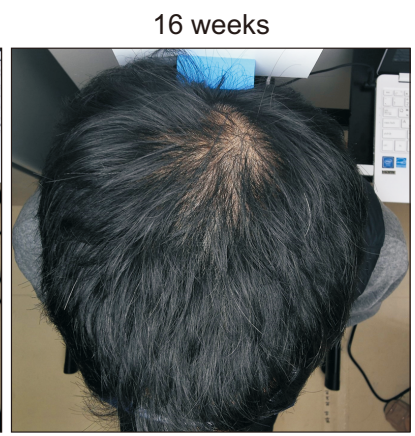

Fig. 7. Photographic images with top side angle of baseline, 8-week, and 16-week of LG Pra'L HGN1treated subject (R-01-48) who was rated as "good improved" by three independent dermatologists.

stimulation have been introduced in medicine for more than 30 years. ${ }^{11,12}$

In the present study, the test group who used LLLT device for 16 weeks showed significant increase in hair density $(18.34 \%, p<0.0001)$ and hair thickness $(16.29 \%, p<$ 0.0001 ) from baseline. Kim et al. ${ }^{8}$ reported similar results with greater hair density in experimental group who used LLLT device for 24 weeks compared to control group who used sham-device. The device used in previous study was composed of LDs with $650 \mathrm{~nm}(4 \mathrm{~mW}, 27$ units) and LED emitting wavelengths of $630 \mathrm{~nm}$ (3.5 mW, 24 units) and $660 \mathrm{~nm}$ (2.5 mW, 18 units), which had light source and energy power different from those of the current study. The mean increase in hair density from baseline was 13.67 counts $/ \mathrm{cm}^{2}$ in our study and which was 17.2 counts $/ \mathrm{cm}^{2}$ in previous study. This difference between primary outcomes may be explained by the difference in duration of the study $(4$ months in the current trial and 6 month in previous trial), length of light emission time, and characteristics of study population. Through this study we 
confirmed that 4 months of observation was sufficient for evaluating the LLLT device in AGA.

Another randomized sham-device controlled study conducted in Thai used helmet type LLLT device containing LDs with $660 \mathrm{~nm}$ of wavelengths (5 mW, 224 units). The result was consistent with our study, showing mean increase of hair density from baseline as 10.21 hairs $/ \mathrm{cm}^{2}$ in experimental group, ${ }^{13}$ which was significantly higher than hair density of sham device-control group.

Although topical minoxidil and oral 5-alpha reductase inhibitors have been corroborated as standard treatment, patients may be discouraged to use them for various reasons as follow: irritation to scalp, underlying conditions such as liver or kidney dysfunction, and adverse events including sexual dysfunction, gynecomastia, and psychiatric disorders. ${ }^{14}$ For those who are unwilling to use these medications, LLLT can be an alternative therapeutic option which has virtually no adverse effect.

This device has a novel array of LD light source. Instead of setting every LD source to support every emitting spot, we made a mirror system that splits one laser source into six emitting spots. With this technology, we could reduce the weight of the device with same potency.

Another progress of this device is the personalized treatment program. Some patients may have relatively severe hair loss in the vertex, while others in the frontotemporal apexes. The device has a wide range of treatment programs to choose according to individual's specific type of AGA.

This study has some limitations. It was designed as a single center study of which study population may be less representative for general population. Although sham device was almost identical to test device in every aspect, minute differences such as temperature or light color may have been recognized by patients. Nevertheless, this study has strength in that the results from double-blind, randomized controlled study have high level of evidence. Our study confirmed the beneficial therapeutic effects of LLLT device with novel array of light source for AGA patients as significant increase of hair density and thickness was observed in experimental group compared to control group.

\section{CONCLUSION}

The home-use LLLT device with novel array of light source and individualized program according to the types of hair loss appears to be an effective and safe treatment modality for both male and female AGA patients.

\section{CONFLICT OF INTEREST}

Yeo-Seon Kwon, Yoon-Young Chang, and Sung-Ho Hong are employees of LG electronics (Seoul, Korea).

\section{FUNDING}

\section{LG Electronics.}

\section{REFERENCES}

1. Cash TF, Price VH, Savin RC. Psychological effects of androgenetic alopecia on women: comparisons with balding men and with female control subjects. J Am Acad Dermatol 1993;29:56875.

2. Han SH, Byun JW, Lee WS, Kang H, Kye YC, Kim KH, et al. Quality of life assessment in male patients with androgenetic alopecia: result of a prospective, multicenter study. Ann Dermatol 2012;24:311-8.

3. Jang WS, Son IP, Yeo IK, Park KY, Li K, Kim BJ, et al. The annual changes of clinical manifestation of androgenetic alopecia clinic in Korean males and females: a outpatient-based study. Ann Dermatol 2013;25:181-8.

4. Tosti A, lorizzo M, Piraccini BM. Androgenetic alopecia in children: report of 20 cases. Br J Dermatol 2005;152:556-9.

5. Rebora A. Pathogenesis of androgenetic alopecia. J Am Acad Dermatol 2004;50:777-9.

6. Avci P, Gupta GK, Clark J, Wikonkal N, Hamblin MR. Low-level laser (light) therapy (LLLT) for treatment of hair loss. Lasers Surg Med 2014;46:144-51.

7. Afifi L, Maranda EL, Zarei M, Delcanto GM, Falto-Aizpurua $L$, Kluijfhout WP, et al. Low-level laser therapy as a treatment for androgenetic alopecia. Lasers Surg Med 2017;49:27-39.

8. Kim H, Choi JW, Kim JY, Shin JW, Lee SJ, Huh CH. Low-level light therapy for androgenetic alopecia: a 24-week, randomized, double-blind, sham device-controlled multicenter trial. Dermatol Surg 2013;39:1177-83.

9. Gupta AK, Foley KA. A critical assessment of the evidence for low-level laser therapy in the treatment of hair loss. Dermatol Surg 2017;43:188-97.

10. Blumeyer A, Tosti A, Messenger A, Reygagne P, Del Marmol V, Spuls PI, et al. Evidence-based (S3) guideline for the treatment of androgenetic alopecia in women and in men. J Dtsch Dermatol Ges 2011;9:S1-57.

11. Avram MR, Leonard RT Jr, Epstein ES, Williams JL, Bauman AJ. The current role of laser/light sources in the treatment of male and female pattern hair loss. J Cosmet Laser Ther 2007;9:27-8.

12. Ghanaat M. Types of hair loss and treatment options, including the novel low-level light therapy and its proposed mechanism. 
South Med J 2010;103:917-21.

13. Suchonwanit P, Chalermroj N, Khunkhet S. Low-level laser therapy for the treatment of androgenetic alopecia in Thai men and women: a 24-week, randomized, double-blind, sham device-controlled trial. Lasers Med Sci 2019;34:110714.

14. Hirshburg JM, Kelsey PA, Therrien CA, Gavino AC, Reichenberg JS. Adverse effects and safety of 5-alpha reductase inhibitors (finasteride, dutasteride): a systematic review. J Clin Aesthet Dermatol 2016;9:56-62.
How to cite this article: Kim JW, Kwon YS, Chang YY, Hong SH, Shin JW, Na JI, Huh CH. Low-level laser therapy with novel array of light source and individualized program for treatment of androgenetic alopecia: a 16-week, randomized, double-blind, sham device-controlled study. Med Lasers 2020; 9:150-158. https://doi.org/10.25289/ML.2020.9.2.150 